\title{
Calorimeter Process Variable Archiving 3823.124-EN-560
}

David Huffman

Jan. 14,2002

\section{Starting, Stopping and Monitoring \\ Starting and stopping the Archiver}

Text with yellow highlight indicates what is typed or printed to the terminal screen.

Additional information and guidelines are available on the online cluster in the /projects/archive area.

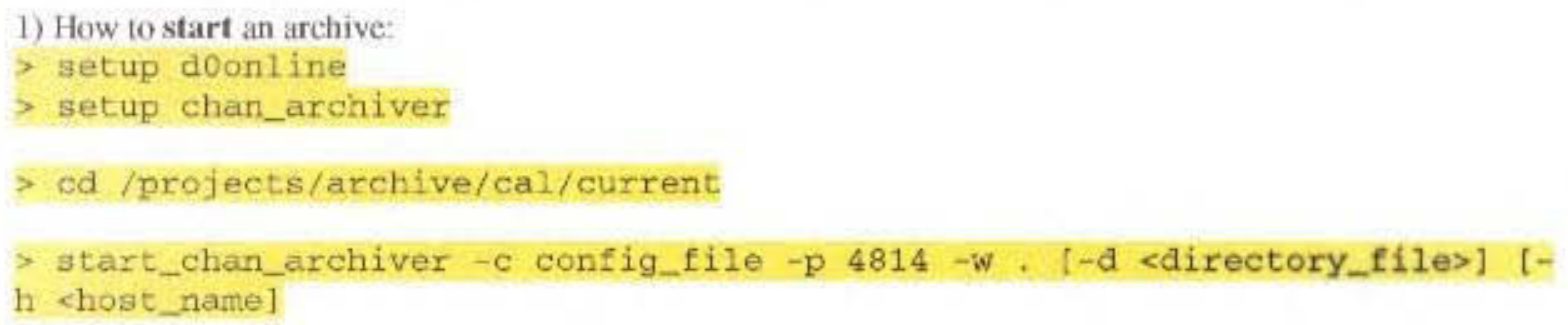

Restarting a stopped archive

NOTE: If the archive is stopped for some reason and it needs to be restarted, the directory file name should be included to prevent a new archive from being created.

For example if the archive with the directory file name dir.20011019-091652 has stop type the following to restart it.

start_chan_archiver -c config_file -p 4814 -w . -d dir.20011019-091652

2) How to stop an archive:

> stop_chan_archiver -p 4814 [-h <host_names]

NOTE: The following will be done by the archive manager.

3) To move data from ./current to Jprevious

This would be done by the person in charge of maintaining the archive with making the weekly file directories and preparing files for storage.

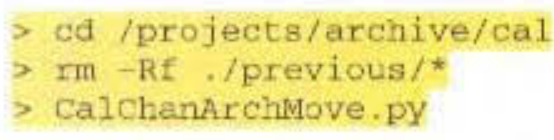

4) To copy, tar and gzip data from /previous to scratch Check that you have directory /doolcluster/scratch/archive/cal ... if not, create it !

$>$ cd /projects/archive/cal

$>$ CalchanArchcopy.py

Scripts to move archives from current to previous and to scratch areas are now in $\$ C H A N \_A R C H I V E R \_D I R / s c r i p t s$ area and can be used via aliases like $>$ chan_arch_move <det> - move archive files from current to previous directories $>$ chan_arch_copy <det>-copy archive files from previous to $/$ doolcluster/scratch/archive/ $<$ det $>/$ dir_<det_ xxxxx area and create tar.gz and catalog files to be exported to SAM

$>$ chan_arch cormpress <det>-will make tar.gz/catalog files in previous directory without copying them into scratch. Will keep only tar.gz and catalog files

Here <det> is the name of your group, like smt,cal,.. 


\section{Calorimeter Process Variable Archiving 3823.124-EN-560}

David Huffman

Jan. 14, 2002

Here are the steps I have been using to maintain weekly archives.

- Friday morning I stop the archiver, wait for it to finish writing data (the lock file will be removed from the directory).

- Move the current archive information to my PC via FTP.

- Remove all previous archive information in the Previous directory.

- Move the current archive into the previous directory

- Start a new archive

- Burn a CDROM of the Archive

- Copy the current archive to d0mino/pri_root/829/d/com/Huffman/dir_*** 


\section{Calorimeter Process Variable Archiving 3823.124-EN-560}

David Huffman

Jan. 14,2002

\section{Monitoring the process}

There are two ways to check if the Calorimeter Archiver is running, either through the WEB based front end or directly from a command line.

Once the Archiver is running it can be monitored from a WEB page at the following address. http://d0ol03.fnal.gov: $4814 /$

NOTE: This only works with a browser launched from the online machine running the archiver.

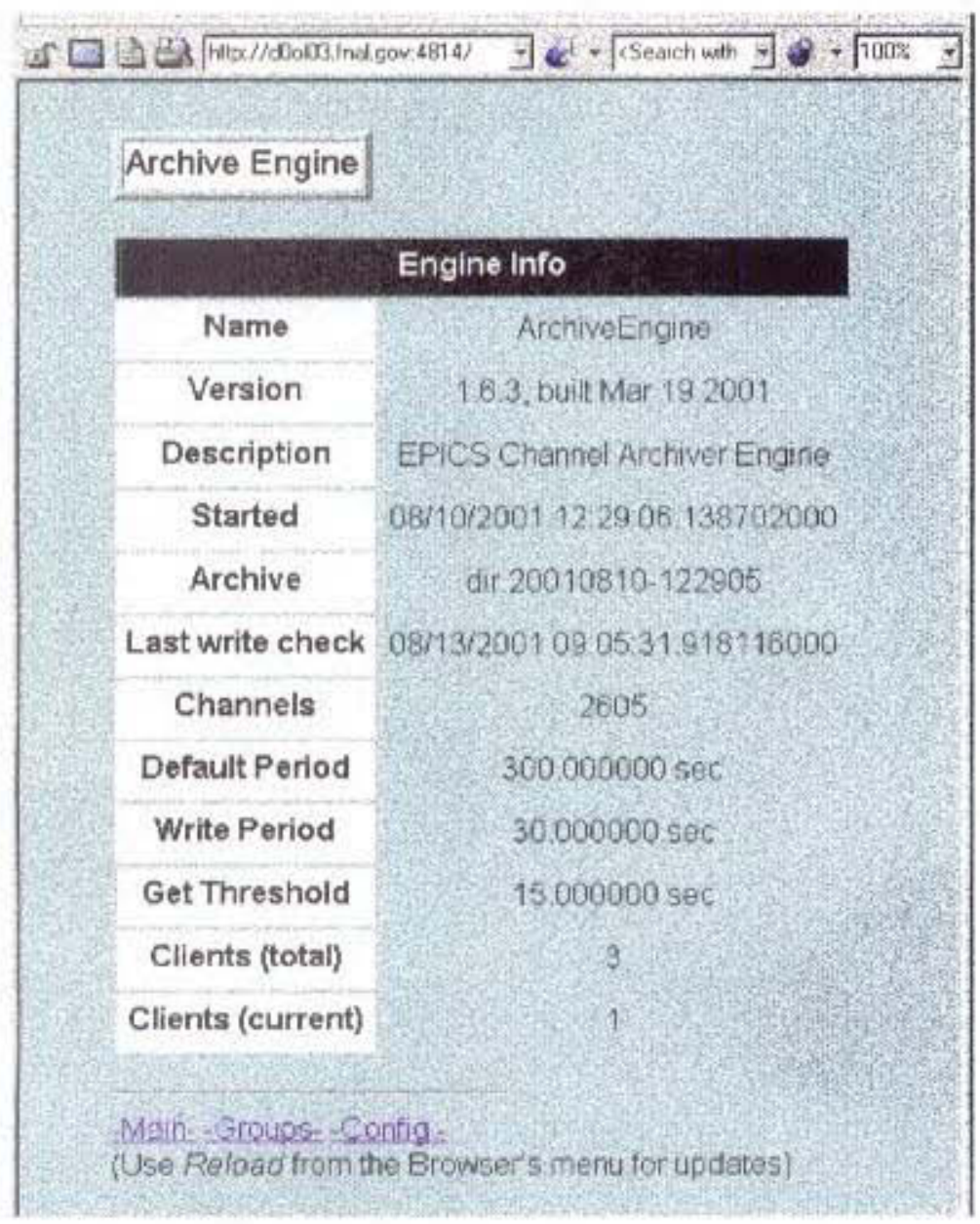

Each time the browser is reloaded there should be an update reported in the Last write check field. You might have to wait a few minutes to see the update. Calorimetery currently takes readings every $(300 \mathrm{sec}$.) 5 minutes. 


\section{Calorimeter Process Variable Archiving 3823.124-EN-560}

David Huffman

Jan. 14, 2002

The second method to verify the Archiver is running is to issue this command from a Linux cluster machine. For example running from $\mathrm{d} 0 \mathrm{ol} 03$ issue the following command. $>$ ps -ef. | grep -i arch

This will return a list of all archivers currently running. The list might look something like this..

\begin{tabular}{|c|c|c|c|c|c|c|c|}
\hline dosmt & 21633 & 1 & 1 & Axyog & $?$ & $03: 43: 04$ & /abusr/produets/chan_ereliver/vi \\
\hline dosmit: & 21634 & 21633 & 0 & Augo3 & ? & $00: 00: 15$ & /dousr/products/chan arebiver/vi \\
\hline dosent & 21635 & 21634 & 2 & Aug03 & 7 & $06: 43: 06$ & /douer/products/chan arehiver/vi \\
\hline sirotenk & 22656 & 1 & 0 & Aug09 & $?$ & $00: 36: 28$ & Gause/proqucte/ehan_archawex/vi \\
\hline \#ir rotenk & 22675 & 22656 & 0 & Aug09 & 7 & $00: 00: 09$ & /atuse/producte/chan_archivier/v] \\
\hline nirotenk & 22676 & 22675 & 0 & $\operatorname{Aug} 09$ & 7 & $00 \div 01: 14$ & /abuse/products/ehan_archiver/vl \\
\hline dorad & 23188 & 1 & 0 & Aas09 & $?$ & $00: 31.47$ & Kabues/products/chan_arekdver /v] \\
\hline dorad & 13189 & 13138 & 0 & Aug09 & 7. & 0.06 & ier; producteichan archives iva \\
\hline dorad & 13190 & 13189 & 1 & Aug09 & ? & $01: 25: 42$ & 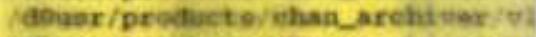 \\
\hline hutfman & 1265 & 1 & 0 & $\operatorname{Aug} 10$ & 2 & $00: 25: 23$ & /aOusr/prebiets/ehan archives/vi \\
\hline hatfman & 1766 & 1765 & 0 & Auglo & 7 & $00: 00: 03$ & /dousr/producte/chen_arebiver/vi \\
\hline huffran & 1767 & 1766 & 0 & Aug10 & 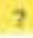 & $00: 24: 53$ & 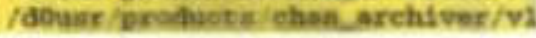 \\
\hline
\end{tabular}

ps auxwo I grep - i huftman

This will display all the information pertaining to the jobs running by user huffman.

Currently I will be starting and stopping the Archiver so the job will be associated with my name. Whoever runs the job will have this privilege. Below is an example of a running archive.

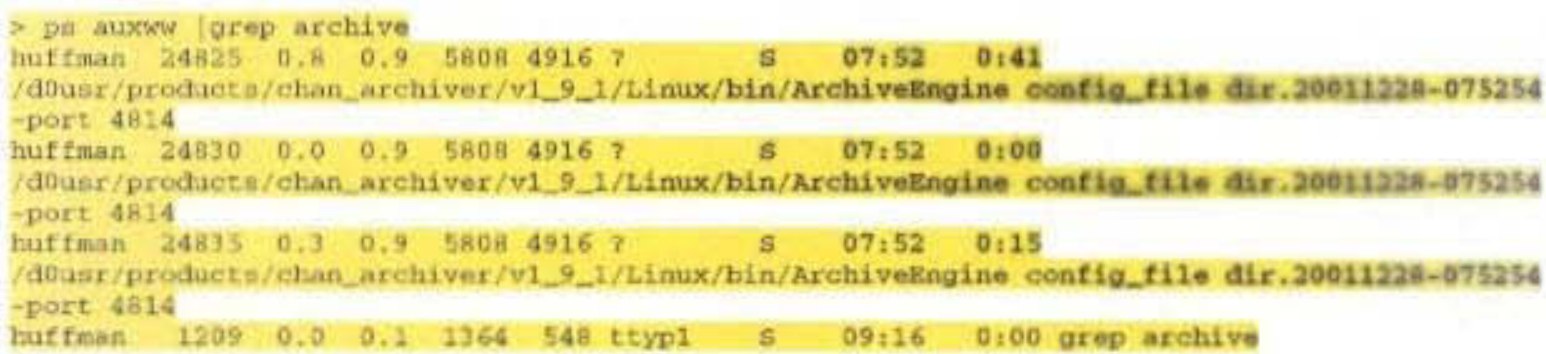

An indicator has been added to the Power Supply GUI to show that the Archiver is running. It appears at the bottom left next to the Reconnect button. The text appears in green while it is running and turns red if it has stopped. 